\title{
SUR UN THÉORÈME DE M. POINCARÉ, RELATIVEMENT AU MOUVEMENT D'UN SOLIDE PESANT
}

\author{
PAR \\ ED. HUSSON \\ à RENNES. \\ Introduction.
}

1. Parmi les résultats remarquables obtenus par M. Poincaré à l'aide des solutions périodiques des équations de la dynamique se trouve le suivant:

Pour qu'il existe, dans le mouvement d'un corps solide pesant autour d'un point fixe, une intégrale première algébrique ne se réduisant pas à une combinaison des intégrales classiques, il est nécessaire que l'ellipsö̈de d'inertie relatif au point de suspension soit de révolution.

La démonstration de M. Poincaré suppose que le produit $\mu$ du poids du corps solide par la distance du centre de gravité au point de suspension est très petite; cependant on l'étend de suite à toutes les valeurs de $\mu$.

La position $d u$ solide étant définie par le système différentiel d'EuLen à l'aide des variables habituelles $p, q, r, \gamma, \gamma^{\prime}, \gamma^{\prime \prime}$, si on remplace $\gamma, \gamma^{\prime}, \gamma^{\prime \prime}$, par $\frac{\lambda}{\mu} \gamma, \frac{\lambda}{\mu} \gamma^{\prime}, \frac{\lambda}{\mu} \gamma^{\prime \prime}$, le système différentiel n'est pas altéré et il dépend du paramètre $\lambda$ qui s'est simplement substitué à la constante $\mu$.

Si le système différentiel initial admet, pour une valeur numérique particulière de $\mu$, une intégrale algébrique, le système transformé admettra une intégrale algébrique pour toutes les valeurs de $\lambda$ et en particulier pour $\lambda$ assez petit, or pour $\lambda$ assez petit la démonstration de M. PoIncaré est applicable.

L'extension précédente est au contraire impossible, en général, si les intégrales premières envisagées sont simplement uniformes au lieu d'être algébriques.

2. Je me propose d'indiquer, pour le théorème rappelé, une démonstration indépendante de celle de M. Poincaré. 
Au point de vue des idées directrices, la méthode employée peut être rapprochée de celles utilisées par M. Poincaré dans l'étude du problème des trois corps et par M. Painlevé dans l'étude des équations différentielles du second ordre dont l'intégrale générale est uniforme.

Le résultat est établi pour toutes les valeurs du poids du corps pesant, pour toutes les positions du centre de gravité, mais en supposant que les conditions initiales sont quelconques.

Lorsque les conditions initiales sont particulières il se peut qu'il existe une intégrale algébrique nouvelle; M. HEss ${ }^{1}$ en a signalé un exemple pour un solide de configuration spéciale, M. STAUDE a mis en évidence l'existence de rotations uniformes autour d'axes invariables dans l'espace pour un solide général.

\section{Transformation préliminaire du système différentiel.}

3. Les équations différentielles du mouvement d'un solide pesant autour d'un point fixe $O$ peuvent toujours être ramenés à la forme,

$$
\begin{cases}A \frac{d p}{d t}=(B-C) q r+y_{0} \gamma^{\prime \prime}-z_{0} \gamma^{\prime}, & \frac{d \gamma}{d t}=r \gamma^{\prime}-q \gamma^{\prime \prime}, \\ B \frac{d q}{d t}=(C-A) r p+z_{0} \gamma-x_{0} \gamma^{\prime \prime}, & \frac{d \gamma^{\prime}}{d t}=p \gamma^{\prime \prime}-r \gamma \\ C \frac{d r}{d t}=(A-B) p q+x_{0} \gamma^{\prime}-y_{0} \gamma, & \frac{d \gamma^{\prime \prime}}{d t}=q \gamma-p \gamma^{\prime}\end{cases}
$$

Les axes $O x, O y, O z$, liés au solide, sont les trois axes de l'ellipsoíde d'inertie relatif au point de suspension, $x_{0}, y_{0}, z_{0}$, sont les coordonnées du centre de gravité $G$.

En adoptant la forme précédente des équations différentielles on suppose $\frac{m g}{O G}=\mathrm{I}$, ce qui revient ̀̀ adopter des unités convenables ou à imaginer que $\gamma, \gamma^{\prime}, \gamma^{\prime \prime}$, sont les produits des cosinus directeurs des axes liés au solide avec la direction de la pesanteur par la constante $\frac{m g}{O G}$.

1 Hess, Mathematische Annalen t. 37; Nexrassorf, Mathematische Annalen t. 47.

'Staude, Journal de Crelle t. II 3 ; Krein et Sommerfeld, Théorie des Kreisels t. II; Levi. Crita, Rendiconti dei Lincei, 190 I et 1905 et Prac matematyczno-fizycznych, (Varsoyie) t. I7. 
Au lieu de conserver les variables $p, q, r, \gamma, \gamma^{\prime}, \gamma^{\prime \prime}$, nous introduirons de nouvelles fonctions choisies de telle façon que les deux premières équations du système (I), par exemple, soient remplacées par deux équations différentielles pour lesquelles les variables soient séparées.

Posons,

$$
\begin{aligned}
& y_{1}=a p+i b q \\
& y_{2}=a p-i b q
\end{aligned}
$$

$a$ et $b$ étant deux constantes, $i$ désignant le symbole $\sqrt{-\mathrm{I}}$.

Les deux premières équations du système ( $\mathrm{I}$ ) sont remplacées par;

$$
\begin{aligned}
& \frac{d y_{1}}{d t}=r\left[\frac{\mathrm{I}}{2 i} \cdot \frac{B-C}{A} \cdot \frac{a}{b}\left(y_{1}-y_{2}\right)+\frac{i}{2} \frac{C-A}{B} \cdot \frac{b}{a}\left(y_{1}+y_{2}\right)\right]+\cdots, \\
& \frac{d y_{2}}{d t}=r\left[\frac{\mathrm{I}}{2 i} \frac{B-C}{A} \cdot \frac{a}{b}\left(y_{1}-y_{2}\right)-\frac{i}{2} \cdot \frac{C-A}{B} \cdot \frac{b}{a}\left(y_{1}+y_{2}\right)\right]+\cdots
\end{aligned}
$$

Ces équations seront respectivement indépendantes de $y_{2}$ et $y_{1}$ sous la condition unique,

$$
\frac{a^{2}}{A(A-C)}=\frac{b^{2}}{B(B-C)}
$$

Nous sommes donc amenés à effectuer le changement de fonctions,

$$
\begin{aligned}
& y_{1}=\sqrt{A(A-C)} \cdot p+i \sqrt{B(B-C)} \cdot q \\
& y_{2}=\sqrt{A(A-C)} \cdot p-i \sqrt{B(B-C)} \cdot q .
\end{aligned}
$$

Pour que l'on puisse substituer la recherche de $y_{1}$ et $y_{2}$ à celle de $p$ et $q$ il est évidemment nécessaire et suffisant que les deux relations précédentes permettent de calculer $p$ et $q$ à l'aide de $y_{1}$ et $y_{2}$, ce qui exige que les deux différences $(A-C)$ et $(B-C)$ soient différentes de zéro.

Pour faciliter les calculs, et, d'une façon plus précise, dans le même but de séparation de variables, nous substituerons à la recherche de $\gamma$ et $\gamma^{\prime}$ celle des deux fonctions,

$$
z_{1}=\gamma+i \gamma^{\prime}, \quad z_{2}=\gamma-i \gamma^{\prime}
$$

Enfin pour faire disparaître les imaginaires nous changerons $t$ en $-i t$.

Le mouvement $d u$ solide pesant sera défini, à l'aide des variables $y_{1}, y_{3}$, $z_{1}, z_{2}, r$ et $\gamma^{\prime \prime}$, par le système différentiel, 


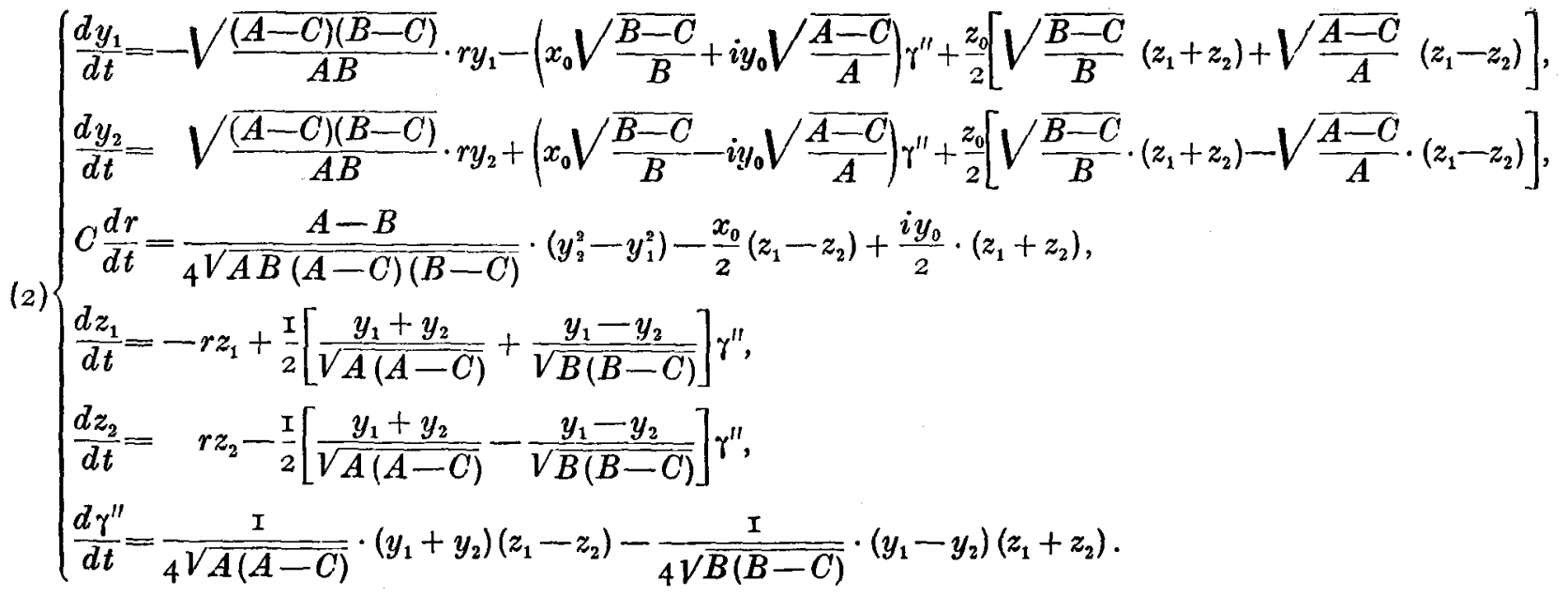

Le système (2) admet les trois intégrales premières classiques des forces vives, des aires et des cosinus directeurs. Ces intégrales s'écrivent,

$$
\begin{aligned}
& \frac{\left(y_{1}+y_{2}\right)^{2}}{4(A-C)}-\frac{\left(y_{1}-y_{2}\right)^{2}}{4(B-C)}+C r^{2}-\left[x_{0}\left(z_{1}+z_{2}\right)-i y_{0}\left(z_{1}-z_{2}\right)+2 z_{0} \gamma^{\prime \prime}\right]=\text { const. } \\
& \begin{array}{c}
\frac{A}{2 \sqrt{A(A-C)}} \cdot\left(y_{1}+y_{2}\right)\left(z_{1}+z_{2}\right)-\frac{B}{2 \sqrt{B(B-C)}} \cdot\left(y_{1}-y_{2}\right)\left(z_{1}-z_{2}\right)+2 C r \gamma^{\prime \prime}=\text { const. } \\
z_{1} z_{2}+\gamma^{\prime \prime 2}=\text { const. }
\end{array}
\end{aligned}
$$

\section{Exposé de la méthode.}

4. Pour déterminer les intégrales premières algébriques du système différentiel (2), remplaçons $y_{1}, z_{1}, z_{2}, \gamma^{\prime \prime}$, par $\lambda y_{1}, \lambda z_{1}, \lambda z_{2}, \lambda \gamma^{\prime \prime}$, sans changer $y_{2}$ et $r$, en désignant par $\lambda$ un paramètre arbitraire. Nous obtenons le système différentiel,

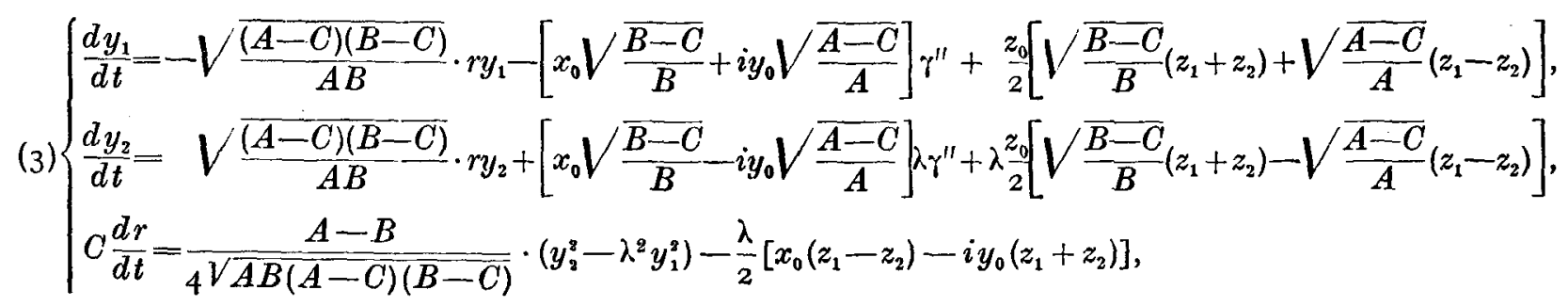


(3) $\left\{\begin{array}{l}\frac{d z_{1}}{d t}=-r z_{1}+\frac{\mathrm{I}}{2}\left[\frac{\lambda y_{1}+y_{2}}{\sqrt{A(A-C)}}+\frac{\lambda y_{1}-y_{2}}{\sqrt{\overline{B(B-C)}}}\right] \gamma^{\prime \prime}, \\ \frac{d z_{2}}{d t}=r z_{2}-\frac{I}{2}\left[\frac{\lambda y_{1}+y_{2}}{\sqrt{A(A-C)}}-\frac{\lambda y_{1}-y_{2}}{\sqrt{B(B-C)}}\right] \gamma^{\prime \prime}, \\ \frac{d \gamma^{\prime \prime}}{d t}=\frac{\mathrm{I}}{4 \sqrt{A(A-C)}}\left(\lambda y_{1}+y_{2}\right)\left(z_{1}-z_{2}\right)-\frac{\mathrm{I}}{4 \sqrt{\overline{B(B-C)}}} \cdot\left(\lambda y_{1}-y_{2}\right)\left(z_{1}+z_{2}\right) .\end{array}\right.$

Le système (3) admet les intégrales premières algébriques,

(4) $\left\{\begin{array}{c}\frac{\left(\lambda y_{1}+y_{2}\right)^{2}}{A-C}-\frac{\left(\lambda y_{1}-y_{2}\right)^{2}}{B-C}+{ }_{4} C r^{2}-4 \lambda\left[x_{0}\left(z_{1}+z_{2}\right)-i y_{0}\left(z_{1}-z_{2}\right)+2 z_{0} \gamma^{\prime \prime}\right]=h_{1}, \\ \frac{A}{2 \sqrt{A(A-C)}} \cdot\left(\lambda y_{1}+y_{2}\right)\left(z_{1}+z_{2}\right)-\frac{B}{2 \sqrt{B(B-C)}} \cdot\left(\lambda^{\prime} y_{1}-y_{2}\right)\left(z_{1}-z_{2}\right)+{ }_{2} C r \gamma^{\prime \prime}=h_{2}, \\ z_{1} z_{2}+\gamma^{\prime \prime 2}=h_{3},\end{array}\right.$

$h_{1}, h_{2}, h_{3}$, étant trois constantes arbitraires que nous pouvons supposer indépendantes de $\lambda$.

5. Les seconds membres des équations (3) sont holomorphes pour $\lambda=0$, par suite pour $\lambda$ assez petit ces équations admettent, d'après une généralisation du théorème de $\mathrm{CAUCHY}{ }^{1}$ une solution développable suivant les puissances croissantes et positives de $\lambda$.

Pour déterminer cette solution générale, ou du moins les premiers termes des développements, nous n'introduirons pas le temps $t$; nous exprimerons $y_{1}, y_{2}, z_{1}, z_{2}, \gamma^{\prime \prime}$, en fonction de $r$. Ceci est toujours possible, sauf dans le cas où $r$ est une constante, c'est à dire dans le cas connu de Lagranger.

Les relations (4), correspondant aux intégrales premières elassiques, donnent $y_{2}, z_{1}, z_{2}$, en fonction de $y_{1}, \gamma^{\prime \prime}, r$, et le système différentiel (3) peut être remplacé par un système de deux équations du premier ordre qui déterminent $y_{1}(r)$ et $\gamma^{\prime \prime}(r)$. On formerait facilement ce système pour la valeur générale de $\lambda$, les valeurs de $\frac{d y_{1}}{d r}$ et $\frac{d \gamma^{\prime \prime}}{d r}$ sont algébriques en $h_{1}, h_{2}, h_{3}, \lambda, y_{1}, \gamma^{\prime \prime}, r$, et sont holomorphes pour $\lambda=0$.

6. Soit pour le système (2),

$$
f\left(y_{1}, z_{1}, z_{2}, \gamma^{\prime \prime}, y_{2}, r\right)=\text { const. ..., }
$$

une intégrale première algébrique, indépendante du temps, et distincte des intégrales classiques.

* Poincart, Méthodes nouvelles de la Mécanique Céleste t. I. 
Le système (3) admet, quel que soit $\lambda$, l'intégrale première algébrique et indépendante du temps,

$$
f\left(\lambda y_{1}, \lambda z_{1}, \lambda z_{2}, \lambda \gamma^{\prime \prime}, y_{2}, r\right)=\text { const. }
$$

Si l'on y substitue les expressions de $y_{2}, z_{1}, z_{2}$, tirées des relations (4), on en déduit que le système des deux équations du premier ordre donnant $y_{1}(r)$ et $\gamma^{\prime \prime}(r)$ admet une intégrale première de la forme,

$$
F\left(h_{1}, h_{2}, h_{3}, \lambda, y_{1}, \gamma^{\prime \prime}, r\right)=\text { const. }
$$

$F$ étant algébrique par rapport à chacune des lettres $h_{1}, h_{2}, h_{3}, \lambda, y_{1}, \gamma^{\prime \prime}, r$.

L'expression $F$ ne peut être indépendante de $y_{1}$ et $\gamma^{\prime \prime}$ sinon, ou bien $r$ serait une constante et l'on se trouverait dans le cas de LAGRANGE, ou bien $F$ serait indépendante de $y_{1}, \gamma^{\prime \prime}, r$, et par suite l'intégrale première $f$ se réduirait à une combinaison algébrique des intégrales classiques.

On peut toujours multiplier l'expression $F$ par une puissance de $\lambda$ choisie de telle sorte que la fonction $F$ de $\lambda$ n'admette le point $\lambda=0$ ni comme zéro ni comme pôle. La fonction algébrique $F$ de $\lambda$ est alors développable, dans le domaine de la valeur $\lambda=0$, suivant les puissances croissantes et positives de $\lambda$ ou de $\lambda^{\frac{1}{p}}$.

Dans ce développement les coefficients des diverses puissances de $\lambda$ sont des fonctions algébriques de $h_{1}, h_{2}, h_{3}, y_{1}, \gamma^{\prime \prime}, r$.

Je puis toujours supposer que pour $\lambda=0, F$ ne se réduise pas à une simple fonction de $h_{1}, h_{2}, h_{3}$.

En effet poussons le développement de $F$ suivant les puissances de $\lambda^{\frac{1}{p}}$ jusqu'au premier terme dont le coefficient ne se réduit pas à une fonction de $h_{1}, h_{2}, h_{3}$.

Soit,

$$
F=\Phi\left(h_{1}, h_{2}, h_{3}, \lambda\right)+\lambda^{\frac{k}{p}} F_{0}\left(h_{1}, h_{2}, h_{3}, y_{1}, \gamma^{\prime \prime}, r\right)+\lambda^{\frac{k+1}{p}} . F_{1}\left(h_{1}, h_{2}, h_{3}, y_{1}, \gamma^{\prime \prime}, r\right)+\cdots
$$

En remplaçant $F$ par la différence,

$$
\frac{F-\Phi\left(h_{1}, h_{2}, h_{3}, \lambda\right)}{\lambda^{\frac{k}{p}}},
$$

ce qui revient à retrancher à l'intégrale première $f$ une fonction algébrique des intégrales classiques, nous voyons que l'on peut toujours supposer que l'on ait, 
(5) $\quad F=F_{0}\left(h_{1}, h_{2}, h_{3}, y_{1}, \gamma^{\prime \prime}, r\right)+\lambda^{\frac{1}{p}} F_{1}\left(h_{1}, h_{2}, h_{3}, y_{1}, \gamma^{\prime \prime}, r\right)+\cdots=$ const., $F_{0}$ n'étant pas indépendant de $y_{1}, \gamma^{\prime \prime}$ et $r$.

7. Faisons $\lambda=0$; le système (3) se décompose en les trois systèmes séparés $(6),(7),(8)$,

$$
\frac{d y_{1}}{d t}=-\alpha_{0} r y_{1}+z_{0}\left(\alpha_{1} z_{1}+\alpha_{2} z_{2}\right)-\alpha_{3} \gamma^{\prime \prime}
$$

(7)

$$
\left\{\begin{array}{l}
\frac{d y_{2}}{d t}=\alpha_{0} r y_{2} \\
\frac{d r}{d t}=\frac{A-B}{4 A B C \alpha_{0}} \cdot y_{2}^{2}
\end{array}\right.
$$

$$
\left\{\begin{array}{c}
\frac{d z_{1}}{d t}=-r z_{1}+\beta_{1} y_{2} \gamma^{\prime \prime} \\
\frac{d z_{2}}{d t}=r z_{2}-\beta_{2} y_{2} \gamma^{\prime \prime} \\
2 \frac{d \gamma^{\prime \prime}}{d t}=y_{2}\left(\beta_{2} z_{1}-\beta_{1} z_{2}\right)
\end{array}\right.
$$

$\alpha_{0}, \alpha_{1}, \alpha_{2}, \alpha_{3}, \beta_{1}, \beta_{2}$ étant des constantes dont les valeurs sont,

(9) $\begin{cases}\alpha_{0}=\sqrt{\frac{(A-C)(B-C)}{A B}}, & \alpha_{3}=x_{0} \sqrt{\frac{B-C}{B}}+i y_{0} \sqrt{\frac{A-C}{A}}, \\ 2 \alpha_{1}=\sqrt{\frac{B-C}{B}+\sqrt{\frac{A-C}{A}},} & 2 \alpha_{2}=\sqrt{\frac{B-C}{B}}-\sqrt{\frac{A-C}{A}}, \\ 2 \beta_{1}=\frac{I}{\sqrt{A(A-C)}}-\frac{I}{\sqrt{B(B-C)}}, & 2 \beta_{2}=\frac{I}{\sqrt{A(A-C)}}+\frac{I}{\sqrt{B(B-C)}} .\end{cases}$

En faisant $\lambda=0$ dans les relations (4), on en déduit que le système des équations différentielles (6), (7), (8), admet les intégrales premières algébriques,

$$
\left\{\begin{array}{c}
\frac{B-A}{(A-C)(B-C)} \cdot y_{2}^{2}+{ }_{4} C r^{2}=h_{1}={ }_{4} C a^{2} \\
\frac{I}{\alpha_{0}}\left(\alpha_{1} z_{1}+\alpha_{2} z_{2}\right) y_{2}+2 C r \gamma^{\prime \prime}=h_{2}, \\
z_{1} z_{2}+\gamma^{\prime \prime 2}=h_{3}
\end{array}\right.
$$

$a$ étant une constante arbitraire remplaçant $h_{1}$ 
On peut le vérifier d'une façon directe et l'on obtient les relations simples suivantes entre les constantes introduites,

$$
\left\{\begin{array}{clrl}
C \beta_{1}=\alpha_{2}\left(\frac{\mathrm{I}}{\alpha_{0}}+\mathrm{I}\right), & C \beta_{2} & =\alpha_{1}\left(\frac{\mathrm{I}}{\alpha_{0}}-\mathrm{I}\right) \\
\alpha_{1} \beta_{1}-\alpha_{2} \beta_{2}=\frac{\mathrm{I}}{2} \frac{B-A}{A}, & 4 \alpha_{1} \alpha_{2}=C \cdot \frac{B-A}{A B} .
\end{array}\right.
$$

8. Dans la suite $y_{1}, y_{2}, z_{1}, z_{2}, \gamma^{\prime \prime}$, désigneront les solutions du système (6), (7), (8), exprimées à l'aide de $r$. Ceci est toujours possible si $r$ n'est pas une constante, c'est à dire si $A$ est différent de $B$.

Comme dans le cas général nous tirons des relations (Io) les valeurs de $y_{2}, z_{1}, z_{2}$, ̀̀ l'aide de $y_{1}, \gamma^{\prime \prime}$ et $r$. Il en résulte d'abord,

$$
\frac{d r}{d t}=\alpha_{0}\left(r^{3}-a^{2}\right)
$$

et le système (6), (7), (8) peut être remplacé par les deux équations du premier ordre,

$$
\left\{\begin{array}{l}
\frac{d y_{1}}{d r}=\frac{-\alpha_{0} r y_{1}+z_{0}\left(\alpha_{1} z_{1}+\alpha_{2} z_{2}\right)-\alpha_{3} \gamma^{\prime \prime}}{\alpha_{0}\left(r^{2}-a^{2}\right)} \\
2 \frac{d \gamma^{\prime \prime}}{d r}=\frac{y_{2}\left(\beta_{2} z_{1}-\beta_{1} z_{2}\right)}{\alpha_{0}\left(r^{2}-a^{2}\right)}
\end{array}\right.
$$

Les seconds membres sont supposés exprimés en fonction de $h_{1}, h_{2}, h_{3}, y_{1}, \gamma^{\prime \prime}, r$, à l'aide des relations (Io) et dépendent algébriquement de ces grandeurs.

9. En faisant $\lambda=0$ dans la relation (5) nous en déduisons que le système (I2) admet l'intégrale première algébrique

$$
F_{0}\left(h_{1}, h_{2}, h_{3}, y_{1}, \gamma^{\prime \prime}, r\right)=\text { const. }
$$

$F_{0}$ ne peut être indépendant de $y_{1}$ et $\gamma^{\prime \prime}$ sinon la fonction $r(t)$ solution du système (6) (7) (8) serait une constante et l'on aurait $A=B$.

L'égalité (I3) montre que les fonctions $y_{1}(r), \gamma^{\prime \prime}(r)$, solution du système (12), et $r$, sont liées par une relation algébrique. En exprimant cette propriété, nous obtiendrons des conditions nécessaires d'existence d'une intégrale première algébrique nouvelle pour le système différentiel (I). 


\section{Etude des conditions nécessaires.}

10. Cherchons à former le système (I2) explicitement.

La première des relations (Io) donne $y_{2}$ en fonction de $r$ et les deux dernières donnent pour $z_{1}$ et $z_{2}$ des expressions irrationnelles en $\gamma^{\prime \prime}$.

Pour éviter les difficultés résultant de cette irrationnalité, nous introduirons, au lieu de $\gamma^{\prime \prime}$, une fonction auxiliaire choisie de telle sorte que le système différentiel devienne rationnel. Cette substitution est d'ailleurs possible d'une infinité de manières.

La seconde des relations (ro) s'écrit,

$$
\alpha_{1} z_{1}+\alpha_{2} z_{2}=\alpha_{0} \cdot \frac{h_{2}-2 C r \gamma^{\prime \prime}}{y_{2}}
$$

et si nous posons,

$$
\alpha_{1} z_{1}-\alpha_{2} z_{2}=u
$$

nous calculerons de suite $z_{1}$ et $z_{2}$ à l'aide de $u$ et $\gamma^{\prime \prime}$, et d'une façon rationnelle.

Les deux expressions $u(r)$ et $\gamma^{\prime \prime}(r)$ sont liées par la relation,

$$
\frac{\alpha_{0}^{2}}{y_{2}^{2}}\left(h_{2}^{2}-{ }_{4} C h_{2} r \gamma^{\prime \prime}+{ }_{4} C^{2} a^{2} \gamma^{\prime \prime 2}\right)-u^{2}=4 \alpha_{1} \alpha_{2} h_{3}
$$

déduite de la troisième des relations (ro).

Si nous prenons comme nouvelle variable,

$$
\alpha_{1} z_{1}-\alpha_{2} z_{2}+\frac{\alpha_{0}}{y_{2}}\left[2 C a \gamma^{\prime \prime}-h_{2} \cdot \frac{r}{a}\right]=v
$$

au lieu de $\gamma^{\prime \prime}$, la relation précédente s'écrira

$$
\left[v+\frac{\alpha_{0} h_{2}}{a} \cdot \frac{r}{y_{2}}\right]^{2}-\alpha_{0}^{2} h_{2}^{2} \cdot \frac{\mathrm{I}}{y_{3}^{2}}+4 \alpha_{1} \alpha_{2} h_{3}={ }_{4} C a \alpha_{0} \cdot \frac{v}{y_{2}} \cdot \gamma^{\prime \prime} .
$$

Les expressions $\frac{d y_{1}}{d r}, \frac{d v}{d r}$, étant rationnelles en $y_{1}$ et $\gamma^{\prime \prime}$, les relations (I4) et ( $\left.\mathrm{I}_{5}\right)$ nous permettront de substituer au système (I2) un système différentiel contenant les expressions $y_{1}(r)$ et $v(r)$ d'une façon rationnelle.

Pour calculer $\frac{d v}{d t}$ on peut utiliser directement la relation (I4), mais il est plus simple de remarquer que l'on a, 


$$
\frac{d u}{d t}=\frac{\alpha_{0}}{y_{2}}\left[2 C a^{2} \gamma^{\prime \prime}-h_{2} r\right]
$$

et par suite,

$$
v=u+\frac{\mathrm{I}}{a} \cdot \frac{d u}{d t}
$$

En se servant du système (6) (7) (8) et des relations (II) on obtient immédiatement,

$$
\frac{d^{2} u}{d t^{2}}=\left(\frac{B-A}{4 A B C \alpha_{0}^{2}} \cdot y_{2}^{2}+r^{2}\right) u
$$

ou bien d'après les relations (ro),

$$
\frac{d^{2} u}{d t^{2}}=a^{2} u
$$

$\Pi$ en résulte, pour définir $v$, l'équation différentielle

$$
\frac{d v}{d t}=a v
$$

et l'on constate que l'équation,

$$
v=0
$$

est une équation intégrale du système (6), (7), (8), ou du système (12).

11. Pour simplifier la première des équations (I2) nous introduirons, au lieu de $y_{1}$, l'expression,

$$
y=y_{1} y_{2}
$$

On a de suite, d'après le système (6), (7)

$$
\frac{d y}{d t}=\alpha_{0} z_{0}\left(h_{2}-2 C r \gamma^{\prime \prime}\right)-\alpha_{3} y_{2} \gamma^{\prime \prime}
$$

ou bien en remplaçant $\gamma^{\prime \prime}$ en fonction de $v$,

$$
\frac{d y}{d t}=\frac{h_{2}}{2 C a^{2}}\left[\frac{z_{0}(B-A)}{2 A B \alpha_{0}} y_{3}^{2}-\alpha_{3} r y_{2}\right]-\frac{\mathrm{I}}{4 C a \alpha_{0}}\left[\alpha_{3} y_{2}^{2}+2 C \alpha_{0} z_{0} r y_{2}\right]\left(v+\frac{h}{v}\right)
$$

$h$ étant une constante arbitraire remplaçant $h_{3}$ et donnée par l'égalité,

$$
h=\frac{A-B}{A B h_{1}}\left(h_{\mathrm{a}}^{2}-h_{1} h_{3}\right) \text {. }
$$

Finalement le système (I2) est remplacé par le système rationnel simple, 
(I6)

$$
\left\{\begin{array}{l}
\frac{d v}{d r}=\frac{a}{\alpha_{0}} \cdot \frac{v}{r^{2}-a^{2}}, \\
\frac{d y}{d r}=a_{1}+a_{2} \frac{r}{\sqrt{r^{2}-a^{2}}}+\left(a_{3} \alpha_{3}+a_{4} z_{0} \frac{r}{\sqrt{r^{2}-a^{2}}}\right)\left(v+\frac{h}{v}\right),
\end{array}\right.
$$

$a_{1}, a_{2}, a_{3}, a_{4}$ étant des constantes numériques non nulles.

Pour arriver aux équations (I6) nous avons supposé essentiellement que les trois différences, $(A-B),(B-C),(C-A)$ sont différentes de zéro.

12. Les fonctions $v(r), y(r)$ s'expriment algébriquement à l'aide de $y_{1}, \gamma^{\prime \prime}, r$; donc, les nombres $A, B, C$ étant tous distincts, pour qu'il existe une intégrale algébrique nouvelle il est nécessaire que les fonctions $v(r), y(r)$ et $r$ soient liées algébriquement.

Or en intégrant le système (I6) on a,

$$
\begin{gathered}
v=k \cdot\left(\frac{r-a}{r+a}\right)^{\frac{1}{2 a_{0}}}, \\
y=a_{1} r+a_{2} \sqrt{r^{2}-a^{2}}+\int\left(a_{3} \alpha_{3}+a_{4} z_{0} \cdot \frac{r}{\sqrt{r^{2}-a^{2}}}\right)\left(v+\frac{h}{v}\right) d r
\end{gathered}
$$

$k$ étant une constante arbitraire.

11 en résulte que les fonctions,

$$
\begin{gathered}
w=\left(\frac{r-a}{r+a}\right)^{\frac{1}{2 \alpha_{0}}}, \\
z=\int\left(a_{3} \alpha_{3}+a_{4} z_{0} \frac{r}{\sqrt{r^{2}-a^{2}}}\right)\left(w+\frac{h^{\prime}}{w}\right) d r,
\end{gathered}
$$

$h^{\prime}$ étant une arbitraire, doivent être liées algébriquement avec la variable indépendante $r$.

Je dis que cette condition ne peut être satisfaite que lorsque le centre de gravité $\mathrm{du}$ solide pesant coìncide avec le point de suspension.

Les trois nombres $A, B, C$ étant distincts, le système différentiel initial est symétrique en $p, q, r$ et nous pouvons toujours supposer que le nombre $C$ est compris entre $A$ et $B$. Le nombre $\alpha_{0}$ est alors imaginaire et la fonction $w(r)$ n'est jamais algébrique.

$\Pi$ en résulte que $z(r)$ s'exprime algébriquement à l'aide de $w$ et $r$.

Or les conditions initiales étant arbitraires, la condition précédente est satisfaite quelle que soit la constante arbitraire $h^{\prime}$ et comme $w$ est indépendante de $h^{\prime}$, les deux intégrales,

Acta mathematica. 31. Imprimé le 27 aont 1907. 


$$
\begin{aligned}
& J_{1}=\int\left(a_{3} \alpha_{3}+a_{4} z_{0} \frac{r}{\sqrt{r^{2}-a^{2}}}\right) w d r \\
& J_{3}=\int\left(a_{3} \alpha_{3}+a_{4} z_{0} \frac{r}{\sqrt{r^{2}-a^{2}}}\right) \frac{d r}{w}
\end{aligned}
$$

s'expriment algébriquement à l'aide de $r$ et $w$.

Considérons un plan sur lequel nous représenterons les valeurs prises par la variable indépendante $r$ et faisons décrire au point représentatif de $r$ un contour fermé très petit entourant le point $r=a$. La fonction $w$ est multipliée par une constante et par suite les intégrales,

$$
\begin{aligned}
& J_{1}^{\prime}=\int\left(a_{3} \alpha_{3}-a_{4} z_{0} \frac{r}{\sqrt{r^{2}-a^{2}}}\right) w d r \\
& J_{2}^{\prime}=\int\left(a_{3} \alpha_{3}-a_{4} z_{0} \frac{r}{\sqrt{r^{2}-a^{2}}}\right) \frac{d r}{w}
\end{aligned}
$$

s'expriment algébriquement à l'aide de $r$ et $w$.

En comparant à $J_{1}$ et $J_{2}$ on en déduit que les quatre intégrales,

$$
\begin{array}{ll}
J_{3}=\alpha_{3} \int w d r, & J_{4}=\alpha_{3} \int \frac{d r}{w}, \\
J_{5}=z_{0} \int \frac{r w}{\sqrt{r^{2}-a^{2}}} d r, & J_{6}=z_{0} \int \frac{r}{w \sqrt{r^{2}-a^{2}}} d r,
\end{array}
$$

doivent s'exprimer algébriquement à l'aide de $r$ et $w$.

\section{Lemme abélien auxiliaire.}

13. Lemme. Soit $P(x)$ une fraction rationnelle, $Q(x)$ un polynome entier en $x$ ou une fraction rationnelle admettant comme pôles simples certains zéros ou pôles de $P(x)$, et,

$$
y(x)=P^{\varkappa}, \quad J(x)=\int \frac{Q}{y} d x,
$$

a étant un nombre irrationnel réel ou un nombre imaginaire. $S i J(x)$ s'exprime algébriquement à l'aide de $y$ et $x$, on a nécessairement,

$$
J(x)=\frac{f(x)}{y(x)}+\text { const. . . , }
$$


Sur un théorème de M. Poincaré, relativement au mouvement d'un solide pesant.

$f(x)$ étant une constante on un polynome entier en, $x$ dont le degré surpasse d'une unité le degré de la fraction rationnelle $Q(x)$.

L'intégrale $J(x)$ est évidemment holomorphe pour toute valeur finie de $x$, distincte d'un zéro ou d'un pôle de $P$.

Soit $x=a$ un zéro d'ordre $m$ de la fraction rationnelle $P$

On a,

$$
y(x)=(x-a)^{m \infty} \cdot y_{1}(x)
$$

$y_{1}(x)$ étant holomorphe et différent de zéro pour $x=a$.

La fonction $\frac{I}{y_{1}(x)}$ est aussi bolomorphe pour $x=a$, puisque $y_{1}(a)$ est différent de zéro; on a donc,

$$
J(x)=\int \frac{Q(x)}{(x-a)^{m x}} \cdot \frac{d x}{y_{1}}=\int \frac{x}{(x-a)^{m x+\check{E}}} \cdot\left[b_{0}+b_{1}(x-a)+b_{2}(x-a)^{2}+\cdots\right] d x,
$$

$E$ étant un entier au plus égal à l'unité.

En intégrant,

$$
J(x)=\frac{\mathrm{I}}{(x-a)^{m a+E-1}} \cdot\left[b_{0}^{\prime}+b_{1}^{\prime}(x-a)+b_{2}^{\prime}(x-a)^{2}+\cdots\right]+C,
$$

$b_{0}^{\prime}, b_{1}^{\prime}, b_{2}^{\prime}, \ldots$ étant des constantes numériques fixées, $C$ une constante arbitraire.

Dans le domaine du point $x=a$, on a d'après l'égalité précédente,

$$
y(x) . J(x)=\varphi(x)+C P^{\alpha},
$$

$\varphi(x)$ étant une fonction holomorphe.

Si la fonction $J(x)$ s'exprime algébriquement à l'aide de $x$ et $y$ il en est de même de $J$ augmentée d'une constante.

Il nous suffit donc de considérer la fonction $J$ pour laquelle la constante $C$ introduite par l'intégration dans le domaine du point $x=a$ est nulle.

Le produit $y(x) . J(x)$ s'exprime algébriquement en $x$ et $y$.

Soit,

$$
y(x) . J(x)=f(x, y)
$$

Dans le domaine du point $x=a$, on a d'après ce qui précède,

$$
f(x, y)=\varphi(x)
$$

Considérons un plan sur lequel nous représenterons les valeurs prises par la variable $x$. Donnons à $x$ une valeur arbitraire $x_{0}$ voisine de $a$, et, faisons décrire au point représentatif de $x$ à partir de $x_{0}$ un nombre quelconque de 
cercles de centre $a$; la fonction holomorphe $\varphi(x)$ garde la même valeur et $y(x)$ prend une infinité de valeurs distinctes en progression géométrique. Or si la fonction algébrique $f\left(x_{0}, y\right)$ dépend effectivement de $y$ elle ne peut garder la même valeur que pour un nombre fini de valeurs de $y$; il faut donc pour que l'égalité (I8) soit possible que la fonction $f$ soit indépendante de $y$, c'est à dire que l'on ait,

$$
y(x) . J(x)=f(x),
$$

$f$ étant une fonction algébrique.

Les seules valeurs finies de $x$ pour lesquelles $f(x)$ peut ne pas être holomorphe sont celles pour lesquelles l'un des deux facteurs $y$ ou $J$ cesse d'être holomorphe. Ces valeurs sont les zéros et les pôles de la fraction rationnelle $P$, exception étant faite pour le zéro particulier $x=a$.

Soit $x=b$ un autre zéro de la fraction rationnelle $P$, on a dans le domaine de ce point, d'après l'égalité ( $\mathrm{I} 7$ ),

$$
f(x)=\varphi_{1}(x)+C_{1} P^{x}
$$

$\varphi_{1}(x)$ désignant une fonction holomorphe, $C_{1}$ étant une constante d'intégration déterminée.

Comme $P^{\alpha}$ admet le point $x=b$ comme point singulier transcendant, cette dernière égalité exige que la constante $C_{1}$ soit nulle.

Si les points $x=a$ ou $x=b$ sont des pôles au lieu d'être des zéros, il suffit dans le calcul initial de changer $m$ en $-m$. On constate que l'égalité (I7) est conservée, par suite le résultat que l'on vient de démontrer pour les zéros de $P$ s'étend aux pôles.

La fonction algébrique $f(x)$ est done holomorphe pour toute valeur finie de $x$. Etudions sa nature pour $x$ infiniment grand.

Posons

$$
x=\frac{\mathrm{I}}{z} .
$$

Si les fractions rationnelles $P$ et $Q$ sont respectivement de degrés $n$ et $n_{1}$ on aura,

$$
y=\frac{\mathrm{I}}{z^{n \alpha}} y_{2}(z), \quad Q=\frac{\mathrm{I}}{z^{m_{1}}} \cdot y_{\mathrm{s}}(z),
$$

$y_{2}$ et $y_{3}$ étant holomorphes et différents de zéro pour $z=0$.

On en déduit,

$$
J(x)=-\int z^{n x-n_{1}-2} \cdot\left(b_{0}+b_{1} z+b_{2} z^{2}+\cdots\right) d z,
$$

ou bien, en intégrant, 
Sur un théorème de M. Poincaré, relativement au mouvement d'un solide pesant.

$$
J(x)=z^{n x-n_{1}-1} \cdot\left(b_{0}^{\prime}+b_{1}^{\prime} z+b_{2}^{\prime} z^{2}+\cdots\right)+C^{\prime},
$$

$b_{0}^{\prime}, b_{1}^{\prime}, b_{2}^{\prime}, \ldots$ étant des constantes numériques, $C^{\prime}$ une constante d'intégration déterminée.

Par suite, on a,

$$
f(x)=y(x) . J(x)=\frac{\mathrm{I}}{z^{n_{1}+1}} \psi(z)+C^{\prime} y_{2}(z) \cdot \frac{\mathrm{I}}{z^{n \alpha}},
$$

$\psi(z)$ étant holomorphe et différent de zéro pour $z=0$.

Comme $z^{n x}$ admet le point $z=0$ comme point singulier transcendant la constante $C^{\prime}$ est nécessairement nulle.

Si $n_{1}$ est supérieur ou au plus égal à - I, le point à l'infini est un pôle d'ordre $\left(n_{1}+\mathrm{I}\right)$ de $f(x)$ et comme $f(x)$ est holomorphe pour toute valeur finie de $x$ il en résulte que cette fonction algébrique est un polynome entier en $x$ de degré $\left(n_{1}+\mathrm{I}\right)$.

Si $n_{1}$ est inférieur à $-I$, le point à l'infini est un zéro pour $f(x)$ et la fonction algébrique $f(x)$ se réduit à un polynome identiquement nul; l'intégrale $J(x)$ ne peut s'exprimer algébriquement à l'aide de $y$ et $x$.

14. Ce lemme peut être généralisé et appliqué lorsque $Q$ est une fonction rationnelle quelconque.

Dans ce cas il est dabord nécessaire que les résidus du quotient $\frac{Q}{y}$, correspondant aux pôles de $Q$ distincts des zéros et pôles de $P$, soient nuls; sinon $J(x)$ admettrait des points singuliers transcendants distincts de ceux de la fonction $y(x)$.

Cette condition étant supposée satisfaite, les seules singularités de la fonction $y(x) . J(x)$ seront des pôles connus et il suffira de reprendre la démonstration en considérant le produit $R(x) \cdot y(x) . J(x), R(x)$ étant un polynome connu choisi de façon que ce produit soit holomorphe pour toute valeur de $x$.

Les conclusions du lemme subsisteront donc, $f(x)$ étant une fraction rationnelle dont le numérateur est de degré déterminé et dont le dénominateur est un polynome connu. 


\section{Démonstration de l'impossibilité.}

15. Revenons aux intégrales $J_{3}, J_{4}, J_{5}, J_{6}$, et exprimons qu'elles se calculent algébriquement à l'aide de $r$ et $w$.

$\mathrm{Si} \alpha_{3}$ est différent de zéro, on a nécessairement d'après le lemme démontré,

$$
\int \frac{d r}{w}=\frac{b r+c}{w}+\text { const., }
$$

$b$ et $c$ étant des constantes.

On en déduit en dérivant,

$$
\frac{I}{w}=\frac{b}{w}-(b r+c) \cdot \frac{w^{\prime}}{w^{2}}
$$

ou bien,

$$
\frac{w^{\prime}}{w}=\frac{a}{\alpha_{0}} \cdot \frac{\mathrm{I}}{r^{2}-a^{2}}=\frac{b-\mathrm{I}}{b r+c},
$$

relation manifestement impossible.

On a donc,

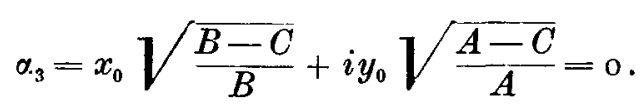

Si l'on reprend la question en échangeant $y_{1}$ et $y_{2}$ on démontrera de même que l'on a,

$$
x_{0} \sqrt{\frac{B-C}{B}}-i y_{0} \sqrt{\frac{A-C}{A}}=0
$$

et il en résulte, par comparaison,

$$
x_{0}=y_{0}=0 \text {. }
$$

Considérons l'intégrale $J_{6}$, on a,

$$
J_{0}=z_{0} \int \frac{r d r}{\left(\frac{r-a}{r+a}\right)^{\frac{1}{2 \alpha_{0}}} \cdot\left(r^{2}-a^{2}\right)^{\frac{1}{2}}}=z_{0} \int \frac{\mathrm{I}}{\left(\frac{r-a}{r+a}\right)^{\frac{1}{2 \alpha_{0}}-\frac{1}{2}}} \cdot \frac{r d r}{r-a} .
$$

Donc si l'on pose,

$$
w_{1}=\left(\frac{r-a}{r+a}\right)^{\frac{1}{2 \alpha_{0}}-\frac{1}{2}}
$$

et si $z_{0}$ est différent de zéro, on a d'après le lemme, 
Sur un théorème de M. Poincaré, relativement au mouvement d'un solide pesant. . 87

et en dérivant,

$$
\int \frac{\mathrm{I}}{w_{1}} \cdot \frac{r d r}{r-a}=\frac{b r+c}{w_{1}}+\text { const. }
$$

ou bien

$$
\frac{r}{r-a}=b-a\left(\frac{\mathrm{I}}{\alpha_{0}}-\mathrm{I}\right) \cdot \frac{b r+c}{r^{2}-a^{2}}
$$

$$
(r+a)[(\mathrm{I}-b) r+a b]=a\left(\mathrm{I}-\frac{\mathrm{I}}{\alpha_{0}}\right)(b r+c) .
$$

On en déduit,

$$
b=\mathrm{r}, c=a
$$

et il reste,

$$
a=a\left(\mathrm{I}-\frac{\mathrm{I}}{\sigma_{0}}\right)
$$

ce qui est impossible.

Donc si $A, B, C$ sont distincts il est nécessaire pour qu'il existe une intégrale algébrique distincte des intégrales classiques que l'on ait,

$$
x_{0}=y_{0}=z_{0}=0
$$

ce qui établit le résultat.

16. On peut appliquer les raisonnements et les calculs indiqués à l'étude des conditions ou domaines d'existence des intégrales premières uniformes; la seule difficulté est d'obtenir une représentation précise et complète du domaine d'existence d'une fonction uniforme de plusieurs variables.

On peut aussi lorsque $A, B, C$, sont distincts, rechercher dans quelles conditions initiales particulières il peut exister une intégrale algébrique nouvelle. On rencontre de suite les relations possibles correspondant aux cas signalés par M. Hess et par M. Staude.

17. Lorsque l'ellipsoïde d'inertie relatif au point de suspension est de révolution, c'est à dire lorsque l'on a, par exemple, .

$$
A=B
$$

on sait que $\mathrm{M}^{\ominus}$ Kovalevsky a obtenu une quatrième intégrale algébrique, pour un solide de configuration spéciale.

On avait espéré que ce résultat remarquable pouvait être généralisé.

Pour étudier ce problème on peut, d'un grand nombre de manières, introduire un paramètre dans le système différentiel. Tous ces paramètres ne conviennent pas également et l'intérêt d'une démonstration directe du théorème 
de M. Poincaré consiste en ce que l'on sépare les procédés qui ne conduisent pas au théorème de M. Poincart. Quelles que soient les recherches auxiliaires dont on les entoure, ces procédés ne peuvent évidemment être utilisés pour aborder le fond de la question.

Pour $A=B$, les nouvelles variables introduites prennent une forme simple, on peut poser,

$$
\begin{array}{ll}
y_{1}=p+i q, & y_{2}=p-i q, \\
z_{1}=\gamma+i \gamma^{\prime}, & z_{2}=\gamma-i \gamma^{\prime},
\end{array}
$$

mais pour $\lambda=0 r$ devient une constante, et il semble impossible d'obtenir des conditions nécessaires complémentaires si loin que l'on pousse les développements des solutions du système différentiel suivant les puissances de $\lambda$.

La même singularité se présente d'ailleurs si l'on adopte le paramètre $\mu$ utilisé par M. Poincarte.

Si l'on remplace $y_{1}, z_{1}, \gamma^{\prime \prime}$, par $\rho y_{1}, \rho z_{1}, \rho \gamma^{\prime \prime}$, en désignant par $\rho$ un paramètre arbitraire, on obtient un nouveau système différentiel holomorphe ${ }^{1}$ pour $p=0$.

En développant les solutions suivant les puissances de $p$ on arrive, comme je l’ai montré dans un mémoire récent, ${ }^{2}$ aux résultats complets suivants:

Les conditions initiales étant supposées arbitraires, les lettres $A, B, C$, représentant les moments d'inertie ou des nombres positifs quelconques, toute intégrale première algébrique et indépendante du temps du système différentiel définissant le mouvement d'un solide pesant autour d'un point fixe est une combinaison algébrique des intégrales classiques.

Il n'y a exception que dans les cas d'Euler, de Lagrange, et de Me KovaLEVSKY.

Lille le 5 juillet 1906.

1 Lorsque $A$ est différent de $B$ cette propriété est inexacte

- Annales de la Faculté des Sciences de Toulouse (1906) et Thèses présentés à la Faculté des Sciences de Paris (1905). 